\title{
Should a normal thromboelastogram allow us to perform a neuraxial block? A strong word of warning
}

\author{
Charles Marc Samama MD PhD
}

I $\mathrm{N}$ this issue of the Journal, Frölich et al. ${ }^{1}$ describe the use of thromboelastography (TEG) to make a clinical decision regarding whether two pregnant patients with thrombocytopenia were eligible for a neuraxial block. They go on to recommend the use of this device to allow the block. Despite a successful outcome, this proposal has to be strongly discouraged.

TEG is a dynamic viskokinetic monitoring device which is very popular, mainly because it is computerized and easy to interpret. ${ }^{2,3}$ It analyses whole blood coagulation and includes the effects of red blood cells and platelets, as compared to the prothrombin time (PT), activated partial thromboplastin time (aPTT) or fibrinogen which are performed on platelet poor plasma. Clinically, from a subjective point of view, it seems more complete and descriptive than the latter. It starts providing information on coagulation profiles almost immediately, contrary to the PT and aPTT that request more than half an hour to be performed because of centrifugation. ${ }^{3}$

TEG is now available in many operating theatres. Physicians deeply involved in TEG monitoring often proclaim that these monitors are very helpful to make differential diagnoses of coagulopathy and to choose the most adequate blood component to achieve optimal hemostasis. Several studies have supported this opinion. ${ }^{4-6}$

As the TEG is a very attractive tool, some groups have proposed that the thromboelastogram could help in making the decision to perform or not a neuraxial block (epidural or spinal anesthesia). Orlikowsky et al. have compared the TEG and the bleeding time (BT) performed before and six hours after a single oral dose of aspirin $600 \mathrm{mg}$ in a group of eight healthy volun- teers and 12 pregnant women. ${ }^{7}$ Measured TEG variables were unaltered after aspirin although there was a significant prolongation of the bleeding time in both groups. This led the authors to consider that the mild prolongation of BT produced by aspirin had been given undue clinical importance and that the TEG may represent "more readily coagulation function". However, the TEG has always been known to be unaffected by aspirin although aspirin is a potent antiplatelet agent. ${ }^{8}$ Therefore it cannot be used to provide the go ahead before performing an epidural anesthesia in a pregnant patient treated with aspirin.

Furthermore, the TEG has never undergone all the validation procedures which are mandatory for conventional hemostasis tests (intra and inter-observer variability, repeatability, calibrations, quality controls). ${ }^{9}{ }^{10}$ Therefore it cannot be recommended in this specific setting and the TEG should not counterbalance a low platelet count.

Very few epidural or spinal hematomas have been reported in the literature in pregnant thrombocytopenic patients. ${ }^{11}$ Therefore this type of accident is very rare and no test of hemostasis is powerful enough to predict the occurrence of such a dramatic complication. The platelet count has to be taken into consideration when allowing a neuraxial block but, even with this simple test, no cut-off value has been defined convincingly $\left(50,000\right.$ to $80,000 \cdot \mathrm{m}^{-3}$ have been suggested depending on the choice of a spinal or an epidural technique respectively, but no randomized study supports this proposal). ${ }^{11}$

Regarding the very specific concern of neuraxial blocks and spinal hematomas, it is not sure that even a large trial including thousands of patients would give any legitimacy to a biological test, whatever it would

\footnotetext{
From the Département d'Anesthésie-Réanimation, Hôpital Avicenne, Bobigny, France.

Address correspondence to: Dr. Charles Marc Samama, Département d'Anesthésie-Réanimation, Hôpital Avicenne, 125 route de Stalingrad, 93009 Bobigny Cedex, France. Phone: 33-1-48-95-55-91; Fax: 33-1-48-95-55-89; E-mail: cmsamama@invivo.edu
} 
be. ${ }^{12}$ Therefore, while the cases presented proceeded uneventfully, recommendations based on the report of only two patients should be considered with extreme caution.

\section{Un thromboélas-} togramme normal devrait-il permettre de réaliser un bloc neuraxial ? Un sérieux avertissement

Dans le présent numéro du Journal, Frölich et coll. ${ }^{1}$ décrivent l'utilisation du thromboélastogramme (TEG) pour faciliter la prise de décision clinique, à savoir si un bloc neuraxial pouvait être administré à deux patientes enceintes, présentant une thrombocytopénie. Ils ont recommandé l'usage de cet appareil pour décider de la pertinence du bloc. Malgré un résultat couronné de succès, cette recommandation doit être vigoureusement découragée.

Le TEG est un appareil de monitorage viscocinétique dynamique très populaire, surtout parce qu'il est informatisé et produit des données faciles à interpréter. $^{2,3}$ Il analyse la coagulation du sang total et les effets sur les globules rouges et les plaquettes, comparativement au temps de prothrombine (TP), au temps de céphaline activé (TCA) ou de fibrinogène qui sont réalisés avec du plasma pauvre en plaquettes. Nous croyons qu'il offre un portrait clinique plus complet et descriptif que les autres. Il commence presque immédiatement à fournir des profils de coagulation, contrairement au TP et au TCA qui demandent plus d'une demi-heure à cause de la centrifugation.

Le TEG se retrouve maintenant dans de nombreuses salles d'opération. Les médecins qui s'intéressent particulièrement au monitorage avec le TEG disent que ces moniteurs sont très utiles pour poser un diagnostic différentiel de coagulopathie et choisir le meilleur composant sanguin permettant une hémostase optimale. Certaines études confirment cette opinion. ${ }^{4-6}$

Comme le TEG est un outil très attrayant, certains groupes ont pensé que le TEG pourrait aider à prendre la décision de réaliser ou non un bloc neuraxial (anesthésie péridurale ou rachidienne). Orlikowsky et coll. ont comparé le TEG et le temps de saignement (TS) réalisés avant et six heures après l'administration orale d'une dose unique de $600 \mathrm{mg}$ d'aspirine à un groupe de huit volontaires en bonne santé et à 12 femmes enceintes. ${ }^{7}$ Les variables de TEG mesurées n'ont pas été modifiées après l'aspirine, mais on a noté une prolongation significative du temps de saignement chez les patients des deux groupes. Ce résultat a conduit les auteurs à considérer qu'on avait donné trop d'importance clinique à la légère prolongation du TS produite par l'aspirine et que le TEG pouvait représenter la "plus aisément la fonction de coagulation". Cependant, on a déjà observé que le TEG n'était pas modifié par l'aspirine, même si l'aspirine est un antiplaquettaire puissant. ${ }^{8}$ Elle ne pourrait donc être utilisée pour donner le feu vert à une anesthésie péridurale chez une femme enceinte traitée à l'aspirine.

En outre, le TEG n'a jamais subi les épreuves de validation obligatoires pour les tests d'hémostase traditionnels (variabilité intra et inter-observateur, répétabilité, calibrages, contrôles de la qualité) ${ }^{9,10} \mathrm{En}$ conséquence, on ne peut la recommander dans ce cadre spécifique et la TEG ne devrait pas contrebalancer une faible numération plaquettaire. Peu d'hématomes rachidiens ou périduraux ont été rapportés chez des femmes enceintes présentant une thrombocytopénie. ${ }^{11}$ Pour cette raison, ce type d'accident est très rare et aucun test d'hémostase n'est assez puissant pour prédire l'occurrence de cette dramatique complication. La numération plaquettaire doit être considérée dans le choix du bloc neuraxial, mais même avec ce simple test, aucune valeur critique n'a été définie de façon convaincante. On a suggéré de 50000 à 80 $000 \cdot \mathrm{m}^{-3}$ selon l'utilisation respective d'une technique rachidienne ou péridurale, mais aucune étude randomisée ne confirme cette proposition. ${ }^{11}$

En ce qui concerne spécifiquement les blocs neuraxiaux et les hématomes rachidiens, il n'est pas sûr que même un essai important comprenant des milliers de patients donnerait de la légitimité au test biologique, quel qu'il soit. ${ }^{12}$ Donc, même si les cas présentés se sont bien déroulés, des recommandations fondées sur les données de deux patientes seulement devraient être considérées avec une extrême prudence.

\section{References}

1 Frolich $M A$, Gibby G, Mabla $M E$.

Thromboelastography to assess coagulation in the thrombocytopenic parturient (Letter). Can J Anesth 2003; 50: 853 .

2 Hartert $H$. Blutgerinnungstudien mit der thromboelastographic. Eine neuen untersuchungsvergahren. Klin Wochenschr 1948; 16: 257. 
3 Mallett SV, Cox DJ. Thrombelastography. Br J Anaesth 1992; 69: 307-13.

4 Kang $\Upsilon_{G}$, Martin DJ, Marquez J, et al. Intraoperative changes in blood coagulation and thromboelastographic monitoring in liver transplantation. Anesth Analg 1985; 64: 888-96.

5 Spiess BD, Gillies BS, Chandler W, Verrier E. Changes in transfusion therapy and reexploration rate after institution of a blood management program in cardiac surgical patients. J Cardiothorac Vasc Anesth 1995; 9: 168-73.

6 Shore-Lesserson L, Manspeizer HE, DePerio M, Francis S, Vela-Cantos F, Ergin MA. Thromboelastography-guided transfusion algorithm reduces transfusions in complex cardiac surgery. Anesth Analg 1999; 88: 312-9.

7 Orlikowski CE, Payne AJ, Moodley J, Rocke DA.

Thrombelastography after aspirin ingestion in pregnant and non-pregnant subjects. Br J Anaesth 1992; 69: 159-61.

8 Trentalange MJ, Walts LF. A comparison of thromboelastogram and template bleeding time in the evaluation of platelet function after aspirin ingestion. J Clin Anesth 1991; 3: 377-81.

9 Whitten CW, Greilich PE. Thromboelastography: past, present, and future. Anesthesiology 2000; 92: 1223-5.

10 Samama CM. Thromboelastography: the next step (Editorial). Anesth Analg 2001; 92: 563-4.

11 Vandermeulen EP, Van Aken H, Vermylen J. Anticoagulants and spinal-epidural anesthesia. Anesth Analg 1994; 79: 1165-77.

12 Horlocker TT, Wedel DJ, Benzon H, et al. Regional anesthesia in the anticoagulated patient: defining the risks (the second ASRA Consensus Conference on Neuraxial Anesthesia and Anticoagulation). Reg Anesth Pain Med 2003; 28: 172-97. 